\title{
|Atenṇão Primária à Saúde: a construção de um conceito ampliado
}

\author{
|Primary Health Gare: the construetion of an expanded concept
}

\section{Resumo}

A Atenção Primária à Saúde assumiu novo protagonismo no discurso internacional e vêm garantindo seu destaque na politica federal há pelo menos dez anos. As concepções de cunho abrangente ganham espaço no cenário político e acadêmico como modelo a ser seguido pelos gestores e profissionais. O estudo objetiva analisar a produção bibliográfica brasileira acerca do conceito de APS, sobretudo a partir de 1990, e suas convergências (ou não) com o discurso consensuado quanto à definição de APS abrangente. Para tanto, realizou-se uma pesquisa bibliográficano cenário nacional para posterior análise de artigos que abordassem discussão conceitual acerca da APS a partir de 1990 a 2012. Os resultados mostraram um aumento considerável do número de publicações que discutem a APS a partir de 2007, bem como o alinhamento de mais da metade das publicações com o ideário abrangente. Evidenciou-se ainda a concentração de estudos que buscaram analisar a integração da APS a rede assistencial como atributo inerente a este serviço e um número considerável de estudos regionais, com análise da política no cenário das unidades de saúde. Conclui-se, portanto, que não obstante tenha havido aumento do número de publicações sobre o tema, sobretudo nos últimos cinco anos, precisa-se elucidar as reais barreiras que impedem a implementação de uma APS abrangente no território nacional.

\section{Abstraet}

The Primary Health Care assumed new role in international discourse and have ensured their importance in federal politics for at least ten years. Comprehensive conceptions are gaining ground in political and academic landscape as a model to be followed by managers and professionals. The study aims to analyze the Brazilian bibliographic production about the concept of APS, especially since 1990, and its convergence (or not) with the consensual definition of APS comprehensive speech. To do so, we performed a literature search on the national scene for later analysis these papers that approached conceptual discussion of APS from 1990 to 2012.The results showed a considerable increase in the number of publications that discuss the APS from 2007 as well as the alignment of more than half of the publications with the comprehensive ideal. Yet it was evident the concentration of studies seeking to analyze the integration of the APS care network as inherent to this service attribute and a number of regional studies, with analysis of policies in the field of health facilities. The results showed a considerable increase in the number of publications that discuss the APS since 2007. More than half of the publications addressed the concept of PHC comprehensive in its theoretical framework, anchored mostly in attributes documented by Bárbara Starfield and the National Primary Care Policy itself. Yet it was evident the concentration of studies seeking to analyze the integration of the APS care network as inherent to this service attribute and a number of regional studies, with analysis of policies in the field of health facilities. Therefore, it has been an increase in the number of publications on the subject, especially in the last five years, and then we must elucidate the real barriers that prevent the implementation of a comprehensive PHC in the country.

\section{Norhan Sumar' 1 \\ Máreia Cristina Rodrigues Fausto ${ }^{2}$}

\author{
Deseritores: Saúde Coletiva, Atenção Primária á Saúde, Sistema Únieo de \\ saúdle
}

Keywords: Public Health, Primary Health Care, Unified Health System

\author{
${ }^{1}$ Espeeialista e mestrando em Saúde Píbliea [Eseola Nacional de Saúde \\ Pública / FIOCRUZ] \\ 2 Doutora em Saúde Coletiva [Instituto de Medieina Social / UERJ] e
Teenologista em Saúde [Fundactäo Oswaldo Cruz]
}

Para correspondência:

Norhan Sumar

E-mail:norhansumar@gmail.com

Data da Submissão: 14/06/2014 Data do Aceite: 14/06/2014 


\section{Introdução}

É reconhecida a importância assumida pela Atenção Primária à Saúde (APS) nos últimos anos e o protagonismo atribuído a esta para reformas de diversos sistemas de serviços de saúde ao redor do mundo, tanto quanto para consequente superação (ou tentativa) do modelo hospitalocêntrico e predominantemente centrado na doença.

Estudos que apontam para condições de saúde potencialmente melhores em países/regiões com maior número de pessoas assistidas por uma atenção primária consistente ${ }^{1}$, além do sucesso dos sistemas de serviços de saúde com uma "forte" atenção primária referida por Srtarfield ${ }^{2}$, reforçam esse pensamento.

É importante destacar, no entanto, que a APS "forte" e "consistente" enunciada por esses autores agrega uma série de atributos e elementos que a caracterizam como APS abrangente, assim como discutiremos a seguir. Além disso, ressaltamos que alternativas estão em debate há décadas e no bojo deste cenário existem diferentes argumentos, interesses e soluções de atores envolvidos.

Ao longo do último século, elementos importantes influenciaram a concepção de APS que figura o debate atual. A primeira referência à um sistema de saúde hierarquizado, com "centros de saúde primários" como a porta de entrada dos usuários e integrados aos demais níveis de assistência especializada e hospitalar remonta aos anos 1920, com a publicação do Relatório Dawson, elaborado pelo então ministro da saúde inglês Lord Dawson of Penn. Ademais, Viana e Fausto $^{3}$ salientam que os movimentos estadunidenses de Medicina Preventiva e Integral em 1940 e Medicina Comunitária em 1960 também influenciaram as discussões e implementação de políticas de APS nos anos subsequentes.

É factível afirmar que a década de 1970 é o marco da efervescência desse debate. Em um contexto de crise econômica e crítica ao papel do Estado Keynesiano, as soluções e ajustes macroeconômicos difundidos pelo ideário neoliberal implicavam, entre outros elementos, redução do papel do Estado no financiamento e provisão de serviços referentes à política social. A política de saúde não ficou isenta ${ }^{4}$.

No entanto, mesmo diante de um cenário conflituoso, elementos pertinentes à concepção ampliada de APS emergem ao final da década. Entre eles, podemos destacar o consenso na trigésima reunião anual da Assembleia Mundial de Saúde (1977), que destacou o nível de saúde necessário à manutenção das atividades diárias e capacidade de trabalho como uma responsabilidade dos governantes e, no mesmo evento,o lançamentodo movimento Saúde Para Todos no Ano 2000 (SPT 2000). Como efeito desse contexto, foi organizada a Primeira Conferência Internacional Sobre Cuidados Primários de Saúde, em Alma Ata, Cazaquistão (1978), cujo objetivo precípuo fora discutir a promoção da saúde para todos os povos do mundo através da atenção primária como modelo de reforma nos sistemas de serviços de saúde.

Neste sentido, além de afirmar a APS como "parte integral do sistema nacional de saúde", conclui-se que esta seja o "primeiro elemento de um processo de atenção continuada à saúde.", que inclui prevenção, promoção, cura e reabilitação ${ }^{5}$.

Atualmente, a APS abrangente, cunhada nos termos de Alma Ata e aprimorada por diversos autores, ganha destaque nos estudos de Bárbara Starfield, que aponta quatro atributos fundamentais: primeiro contato, longitudinalidade, integralidade e coordenação do cuidado. Além de dois atributos indiretos: cuidado centrado na família e orientado para comunidade.

Mata e Ruiz 6 incluem no escopo da APS abrangente a intersetorialidade, ao considerarem a saúde o resultado de múltiplos fatores, não limitados apenas ás questões biológicas. Desta forma, faz-se necessário a ação de diversos setores da sociedade no enfrentamento de questões que tenham influência para o processo saúde-doença.

$O$ fato é que a concepção abrangente de APS não era a única em voga. No ano seguinte à Conferência de Alma Ata uma conferência organizada em Bellágio (Itália) para discutir a abrangência e o custo das propostas da APS enunciadas em Alma Ata, além das incertezas quanto ao cumprimento das metas estabelecidas no SPT 2000, foi patrocinada pela Fundação Rockfeller e contou com a participação do Banco Mundial, UNICEF, Agência de Desenvolvimento Internacional dos EUA, Fundação Ford e Centro de Pesquisas e Desenvolvimento Internacional do Canadá.

É nesta ocasião que nasce como proposta e como conceito a Atenção Primária à Saúde 
"Seletiva" (APSS), estavacentrada em programas de saúde verticais, desarticulados da ideia de determinação social do processo saúde-doença, focalização e seletividade através de práticas que contemplavam uma cesta básica de serviços, ações de saúde simples e de baixo custo, sobretudo direcionadas aos pobres, entre outros aspectos que negligenciavam o que fora proposta em Alma Ata.

Em um cenário de exacerbação da crise econômica global, essa proposta foi amplamente difundida por parte do Banco Mundial e tais programas seletivos de atenção asaúde-com adesão de organismos de cooperação internacional-foram financiados principalmente nos países periféricos ${ }^{7}$.

No Brasil, reflexos desse movimento podem ser percebidos nas experiências de expansão da cobertura da década de 1980 e, posteriormente através do Programa de Agentes Comunitários de Saúde (PACS) a partir de 1990, dando impulso inicial para o que seria denominado Programa de Saúde da Família (PSF) quatro anos mais tarde.

Cabe reconhecer, entretanto, a influência das experiências alternativas (quiçá contra hegemônicas)ao longo das décadas de 1970 e 1980 para a atenção primária brasileira e para o Movimento Sanitário. Entre elas, podemos citar o Projeto Montes Claros (PMC), Projeto de Medicina Comunitária de Londrina (PMCL), Programa de Atenção Primária Projeto Niterói, entre outros. Princípios como hierarquização, universalização, integralidade, administração democrática e eficiente e regionalização eram permeados pela valorização da abordagem médico-social e da saúde como prática política ${ }^{8}$.

Nos últimos anos, os enunciados da política de APS brasileira vêm ganhando reformulações e aprimoramentos que sugerem abrangência e consonância com a concepção citada por Starfield ${ }^{2}$. A aproximação dos documentos oficiais com os atributos referidos pela autora ganha ainda mais força após a tradução e publicação pelo Ministério da Saúde do livro "Atenção Primária: equilíbrio entre necessidades de saúde, serviços e tecnologia".

A Política Nacional de Atenção Básica, publicada em 2006 e sua revisão em $2011^{9}$ 10, situam a Estratégia de Saúde da Família (ESF) como caminho prioritário para reorganização da Atenção Básica (aqui entendida como termo correlato à Atenção Primária à Saúde). Além disso, trazem como fundamentos da APS brasileira: território adstrito, acesso universal e contínuo, vínculo e adscrição de clientela, coordenação da integralidade, estímulo a participação dos usuários. A atenção básica, segundo o documento, considera o sujeito em sua singularidade e inserção sociocultural, buscando produzir a atenção integral.

Não há dúvidas quanto ao aprimoramento das estratégias e diretrizes da APS brasileira no sentido de torná-la abrangente. Ainda que não se possa garantir que isso se traduza em experiências abrangentes de implementação da política. O fato é que, reforçar o aparato normativo e legal e acompanhar esse processo com a produção acadêmica e tensionamento político, pode contribuir para viabilização de casos exequíveis de APS em termos de qualidade e abrangência.

Não esperamos uma política de APS seja integralmente abrangente ou seletiva. As características que as diferem como propostas são pertinentes para fins didáticos e de compreensão dos processos históricos que culminaram na concepção de APS hegemônica mundial, bem como seus reflexos para o caso brasileiro. A importância de perceber tais diferenças entre essas propostas e a relação com os processos históricos recentes é fundamental para suscitar novas formulações e aprimoramentos.

Pensar em uma APS abrangente no bojo de um sistema de saúde em essência contraditório como o caso brasileiro é ainda mais audacioso. As limitações impostas pela convivência de interesses do mercado da saúde disputam cenário com as contradições inerentes ao setor, que permeiam a luta pela garantia do direito frente àiniquidade na distribuição de recursos e serviços entre as regiões do país, subfinanciamento público, medicamentalização da saúde, incorporação tecnológica, entre inúmeros outros.

A história, aqui entendida como um processo dinâmico e não linear, é permeada por lutas, correlações de força e influências de atores distintos. Nesse sentido," recorrer à história, não significa esgotá-la"11. Não pretendemos esgotar todos os fatos relacionados à construção do conceito e das concepções de APS hoje conhecidas, mas destacar elementos importantes e pertinentes, comumente referidos pelos autores que pesquisam o tema no Brasil. 


\section{Metodologia}

Trata-se de um estudo de revisão da literatura, que buscou investigar a produção acadêmica nacional acerca do conceito de APS e seus distanciamentos e aproximações com os conceitos de APS abrangente e/ou seletiva.

Para resgate da produção, iniciamos a busca a partir de leituras de artigos e livros relacionados ao tema da pesquisa que nos subsidiasse no sentido de definir, segundo análise própria, o que seria APS abrangente ou seletiva. Ademais, a leitura dos documentos de governo, incluindo as duas $\mathrm{PNAB}^{9}{ }^{10}$, com o mesmo interesse de identificar as similitudes do aparato normativo e legal com o discurso abrangente da APS encontrado na literatura.

Há um número crescente de artigos e demais produções acadêmicas que buscam abordar a APS sob diversos aspectos diferentes. Fato este que nos impôs a necessidade indubitável de definirmos um planejamento de busca que garantisse um número plausível de artigos para análise, ao passo que também tratassem dos elementos que aspirávamos discutir.

Pensou-se, a priori, em associar os descritores de atenção primária (Atenção Primária, Atenção Primária à Saúde e Atenção Básica) com descritores que contemplassem o termo "conceito". No entanto, o formulário de descritores utilizado (DECS) contém apenas "Formação de Conceito" como alternativa, o que garantiu recuperar apenas dois artigos na Biblioteca Virtual em Saúde (BVS).

O planejamento de busca manteve-se na BVS por se tratar de uma base de dados que garante recuperar artigos das revistas de relevância nacional e internacional, em quantidade e qualidade admissíveis. Entretanto, mudamos a forma de trabalhar no que diz respeito aos descritores. Ao investigar somente os descritores relacionados à atenção primária, articulados com o operador booleano "OR" e sem combiná-los com outro descritor, recuperamos 2.405 publicações.

Para o tempo em que se propôs concluir o estudo e o número de pessoas envolvidas em sua confecção, entendemos que o número de artigos recuperados era excessivo, o que nos levou a utilização de filtros no sentido de delimitar mais o escopo de artigos que tratassem das questões relacionadas aos nossos objetivos.

Os seguintes filtros foram utilizados:
Ações em saúde e erradicação da pobreza; Atenção Integral a Saúde; Ciência, Tecnologia e inovação; Cooperação Internacional; Gestão, educação e participação; Promoção e vigilância; textos completos; Brasil. Neste contexto, reduzimos o número de publicações recuperadas para trezentos e quatro, das quais trinta eram teses ou dissertações e um trabalho de conclusão de curso latu sensu, que foram excluídos da análise a priori. Definiu-se esta busca como sendo a disparadora para nossa análise da produção bibliográfica.

Cabe ressaltar que os filtros escolhidos para o estudo figuram entre aqueles disponibilizados para buscas no portal da BVS, dos quais excluímos apenas Assistência Farmacêutica, Rede de Serviços de Urgência, Saúde Suplementar, Saneamento Básico e Saúde Ambiental por acharmos incompatíveis com a discussão do presente estudo.

Foram selecionados para leitura completa trinta e cinco artigos. Desses, foram incluídos na discussão vinte nove121314151617181920 21 22 2324252627 28293031323334353637383940 que atenderam aos critérios de inclusão por fazerem discussão conceitual acerca da APS abordando alguns de seus elementos de abrangência ou seletividade. Não há critérios temporais para a inclusão na análise. Identificou-se na busca que a concentração de publicações no Brasil se dá a partir da década de 1990 e acentua-se na década seguinte, como discutiremos a seguir.

\section{Resultados}

\section{Estudos sobre APS em periódicos nacionais: breve caracterização}

Os documentos oficiais brasileiros, sobretudo a partir da PNAB 20069, utilizam um conceito de APS muito próximo ao referenciado por Starfield ${ }^{2}$. Para a autora, a APS de cunho abrangente ancora-se em quatro atributos considerados diretos: porta de entrada preferencial do sistema de saúde, integração com demais níveis de complexidade, coordenação do cuidado pela atenção primária e continuidade da atenção. E ainda de dois atributos indiretos: cuidado centrado na família e orientação para a comunidade. O PSF, bem como referimos anteriormente, passou por um processo de transição de política com características de 
focalização para estratégia de reorientação do modelo assistencial vigente, assumindo caráter abrangente em suas definições e conceitos.

Entretanto, antes de iniciarmos a reflexão acerca dos resultados desta pesquisa, e dadas ascircunstâncias latino-americanas de conformação de seus sistemas de saúde nacionais, sobretudo dos serviços de APS41, lembramos que o caráter universal de uma política não exclui a possibilidade de focalização dentro desta, desde que a focalização seja um meio e não traduza seus princípios ${ }^{42}$. Além disso, cabe ressaltar que independente dos elementos da APS abrangente contidos no aparato normativo e legal da política, não garantem que a mesma seja abrangente na prática.

Os artigos selecionados para o estudo estão distribuídos entre os periódicos mais conceituados do país. Dentre os quais, os três primeiros elencados na Tabela 1, são conceituados como "A Internacional" pela Coordenação de Aperfeiçoamento de Pessoal de Nível Superior (CAPES). Fato que coincide com outros estudos de revisão acerca do tema envolvendo APS21.

\begin{tabular}{|c|c|c|c|c|}
\hline Periódico & $\begin{array}{l}\text { Atenção } \\
\text { Primária }\end{array}$ & $\begin{array}{c}\text { Atenção } \\
\text { Básica }\end{array}$ & $\begin{array}{c}\text { Atenção } \\
\text { Primária } \\
\text { à saúde }\end{array}$ & Total \\
\hline $\begin{array}{l}\text { Cadernos de } \\
\text { Saúde Pública }\end{array}$ & & 2 & 7 & 9 \\
\hline $\begin{array}{l}\text { Revista de } \\
\text { Saúde Pública }\end{array}$ & & & 2 & 2 \\
\hline $\begin{array}{l}\text { Revista } \\
\text { Panamericana } \\
\text { de Salud } \\
\text { Pública }\end{array}$ & 1 & & 2 & 3 \\
\hline $\begin{array}{l}\text { Ciência e } \\
\text { Saúde } \\
\text { Coletiva }\end{array}$ & 1 & 2 & 7 & 10 \\
\hline Outros* & & & 5 & 5 \\
\hline Total & 2 & 4 & 23 & 29 \\
\hline
\end{tabular}

Tabela 1. Textos completos disponibilizados no portal da Biblioteca Virtual em Saúde a partir dos descritores/palavras-chaves: Atenção Primária, Atenção Básica, Atenção Primária à Saúde, porperiódico. 2000 a 2012.

* Entre estes periódicos, temos a Revista Mineira de Enfermagem, Saúde e Sociedade, Cogitare Enfermagem e Cadernos de Terapia Ocupacional da Universidade Federal de São Carlos (UFScar).

Fonte: Biblioteca Virtual em Saúde (http://brasil.bvs.br/, acessado em 20 de junho de 2012).
No que se refere à distribuição regional das publicações, a tendência de concentração de estudos produzidos no eixo Sul-Sudeste e vinculados a instituições de ensino e pesquisa se mantém, assim como evidenciado pelo Gráfico 1 . Aproximadamente metade (15 artigos) do universo de artigos analisados está concentrada em instituições do Rio de Janeiro, São Paulo, Minas Gerais, Rio Grande do Sul e Paraná. Desses, doze são vinculados a instituições de ensino e pesquisa do Rio de Janeiro (6 artigos) e São Paulo (6 - além de um estudo cujos autores estão vinculados á Secretaria Estadual de Saúde). Apenas duas publicações cuja origem seja a região centro-oeste e também duas da região nordeste (destaca-se, porém, um número relevante de publicações oriundas da Universidade Federal da Bahia (5), o que eleva a média de produção da região Nordeste)e uma atribuída ao Ministério da Saúde.

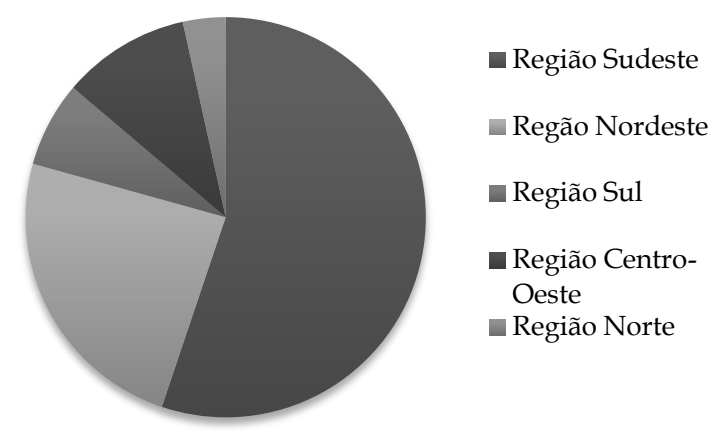

Gráfico 1. Distribuição dos artigos analisados no estudo, segundo região do Brasil, 2000 a 2012.

Fonte: Elaboração própria

Segundo pesquisa realizada por Carvalho e Medina ${ }^{43}$ acerca da produção acadêmica sobre PSF no Brasil, há um elevado número de publicações na região Sudeste e região Nordeste desde 1998. As regiões Norte e Centro-Oeste, segundo as autoras, apresentam uma quantidade incipiente de publicações neste período em relação às demais regiões do Brasil, o que supostamente coincide com a distribuição da oferta de serviços de saúde e número de 
instituições de ensino e pesquisa lotadas nestas.

Neste sentido, dada a importância da produção científica para nortear, entre outras coisas, a formulação de políticas, práticas e fazeres, evidenciamos a necessidade de incentivar a realização de estudos acerca da APS nas regiões fora do eixo Sul-Sudeste. Tanto para entendermos ou apontarmos as limitações impostas por cada cenário para a implantação de uma política complexa como a de APS, quanto os constrangimentos desta implantação em um país continental como o Brasil.

Além disso, cabe o questionamento a respeito do papel dos profissionais de saúde do PSF nos estudos acerca da APS. Poucos estudos estão vinculados aos serviços de saúde diretamente, o que coloca o profissional de saúde como objeto ou amostra do estudo. Nesta perspectiva, consideramos a importância destes atores no processo de transformação das práticas relacionadas a política e, portanto, o potencial para produção de novos estudos que eviscerem aspectos que só podem ser desvelados por aqueles que vivem o seu cotidiano.

Não obstante entre os critérios de exclusão não houvesse nada relacionado ao ano de publicação dos estudos e, portanto, tenhamos recuperado - após a inclusão dos filtros trezentos e quatro artigos publicados entre 1989 e 2013, os trinta e cinco artigos escolhidos para leitura completa tinham suas publicações datadas entre os anos 2000 e 2013, assim como explicita a Tabela 2. Entre os vinte e nove selecionados para análise, vinte e três têm publicação entre 2009 e 2012. Em relação aos seis artigos selecionados para análise publicados antes de 2008, embora um deles tenha sido publicado em 2000, há um salto até o ano de 2006, onde mais um artigo figura a nossa análise, seguidos de dois do ano de 2007 e dois de 2008.

A concentração de artigos selecionados para análise entre os anos de 2008 e 2012 acompanha o total de publicações acerca do tema (após filtragem realizada para o estudo) neste período. A média de publicações anuais entre os anos de 2000 e 2006 foi de 5,4 e a média entre os anos de 2007 e 2012 é de 42,6. Cabe ressaltar ainda o crescimento do número de publicações a partir de 2008, que com 40 artigos publicados, foi superior ao ano de2007 que teve 17. O ápice está entre 2009, 2010 e 2011, com 57, 56 e 56 respectivamente, com queda moderada no ano de2012, com 30 publicações.

\begin{tabular}{|c|c|c|c|}
\hline $\begin{array}{c}\text { Ano de } \\
\text { publicação }\end{array}$ & $\begin{array}{c}\text { Artigos } \\
\text { selecionados } \\
\text { para o } \\
\text { estudo }\end{array}$ & $\begin{array}{c}\text { Todos } \\
\text { os } \\
\text { artigos } \\
\text { da } \\
\text { busca } \\
\end{array}$ & $\begin{array}{c}\text { Média de } \\
\text { Publicações/ano }\end{array}$ \\
\hline 2000 & 1 & 4 & \multirow{3}{*}{5,4} \\
\hline 2006 & 1 & 7 & \\
\hline 2007 & 2 & 17 & \\
\hline 2008 & 2 & 40 & \multirow{5}{*}{42,6} \\
\hline 2009 & 7 & 57 & \\
\hline 2010 & 2 & 56 & \\
\hline 2011 & 8 & 56 & \\
\hline 2012 & 6 & 30 & \\
\hline Total & 29 & $267^{*}$ & \\
\hline
\end{tabular}

*Número total de artigos após a exclusão das teses e dissertações.

Fonte: Biblioteca Virtual em Saúde (http://brasil.bvs.br/, acessado em 18 de junho de 2012).

A causa para a concentração de publicações entre esses anos após a aplicação dos filtros selecionados para o estudo aponta para o possível sucesso na escolha destes elementos, pois é a partir do início da década passada que se inicia a discussão acerca dos conceitos de APS do cenário brasileiro, potencializada a partir da segunda metade da mesma (Gil, 2006).

\section{O conceito abrangente utilizado por autores brasileiros: ascensão da politica nacional como referêneia}

Entre os vinte e nove artigos que foram analisados neste estudo, dezenove deles traziam em seu referencial teórico uma concepção de APS abrangente 121314171922232425262728293031323435 3740 abordando ao menos dois de seus atributos. No cenário brasileiro, cujo Sistema de Serviços de Saúde herda traços históricos de fragmentação, participação maciça da iniciativa privada - tanto no setor de saúde suplementar quanto na prestação de serviços ao SUS -, rebatimentos importantes advindos das medidas do receituário neoliberal, apenas dois dos artigos analisados apresentavam o conceito de APS sob o prisma da focalização. Os demais artigos não faziam referência à algum conceito específico de APS, 
ainda que se referissem aos atributos em determinados momentos.

Ainda neste contexto, mais de um terço deles $^{12} \begin{array}{lllllllllll}13 & 14 & 19 & 22 & 23 & 24 & 26 & 27 & 28 & 31 & 40 \\ \text { apresentavam o }\end{array}$ conceito de Starfield como definidor de uma APS abrangente, e nove131822 24262729313537 39também se reportavam a Política Nacional de Atenção Básica, hoje expandida através do PSF como referência brasileira. A associação entre o conceito de Bárbara Starfield e os fundamentos contidos nos documentos oficiais explicita, ao menos numa primeira análise, tanto o reconhecimento destes atributos como pertinentes ao contexto brasileiro, quanto a referência aos textos oficiais para definição de uma APS abrangente.

Cabe destacar que, em 2002, o Ministério da Saúde publicou e distribuiu entre atores da saúde pública a versão em português do livro PrimaryCare: balancinghealthneeds, servicesandtechnology (Atenção Primária: equilíbrio entre necessidades de saúde, serviços e tecnologia), de Bárbara Starfield, que passoua influenciar tanto a produção ministerial, quanto a produção acadêmica nacional.

Visto que dentre os estudos selecionados para análise, todos esses citados no parágrafo acimaforam publicados entre 2007 e 2011, especulamos a importância do texto da PNAB publicada em 2006 para o reconhecimento conceitual de uma APS abrangente no contexto nacional.

Evidenciamos ainda aqueles autores que se referem ao PSF como modelo de APS abrangente, mencionando entre as referências somente os documentos oficiais. Sete 18252930353739 artigos entre os analisados não citaram publicações internacionais e se referiram a APS brasileira como marco abrangente, no que tange a essência contida nos textos governamentais.

\section{O dilema da integração da rede assistencial}

É reconhecida a turbulência histórica acerca da integração da rede assistencial na saúde pública brasileira, sobretudo no SUS, e sua relação direta com a integralidade da assistência e coordenação do cuidado em saúde em sistemas fundamentados pela APS, assim como enunciam Hartz e Contandriopoulos, (2004). A regionalização e integração preconizadas na NOAS 01/2001, também referidas na PNAB 2011 como Redes de Atenção a Saúde (RAS) são elementos pressionados pelas limitações estruturais da saúde brasileira.

Assim como já referido anteriormente, os países latino-americanos, tradicionalmente permeados por sistemas fragmentados e segmentados, enfrentam dificuldades estruturais para a superação destas barreiras cerceadoras dos atributos da APS abrangente ${ }^{41}$.

Neste sentido, destacamos que o resultado da nossa busca aponta para um interesse em estudar a integração da rede assistencial por parte dos pesquisadores. Entre integração para coordenação do cuidado, integralidade da atenção e garantia de referência a rede especializada, aproximadamente um terço dos estudos (dez) se propunham a analisar os limites e barreiras impostas a este atributo da APS em relação ao sistema de saúde 12131417182230343640 .

Hartz e Contandriopoulos (2004) já apontavam para ganhos promissores no cenário brasileiro acerca da integração como via de integralidade da assistência, sob o ponto de vista ampliado. A garantia da integração da rede assistencial para a consolidação de uma APS abrangente é imprescindível, tanto porque sua articulação com os atributos da coordenação do cuidado e longitudinalidade é inerente.

A análise posterior dos resultados destes estudos é importante para conhecermos os avanços ou estagnações do cenário político e prático, para fomentarmos o debate no sentido de continuar alimentando a reforma sanitária brasileira para consolidação da saúde como direito fundamental.

\section{O micro e o macro: difieuldades para análise da política de forma macro dinâmica}

Quinze14151718202325262833 3436373940 artigos entre os analisados tratavam de estudos loco regionais, cujos objetivos permeiam a análise de unidades de saúde da família e de atenção básica no interior dos seus respectivos municípios. Do universo de artigos explorados, menos de um quarto deles analisaram a política de atenção primária no sentido amplo e de forma macro dinâmica, buscando evidenciar as lacunas na tentativa de implementação dos atributos da APS que figuram a Política orientadora.

Gil (2006) já apontava para esta tendência de concentração de estudos acerca das realidades 
vivenciadas em unidades de saúde e a carência de estudos que contextualizassem a APS no cenário nacional, no modelo de atenção a saúde ou mesmo nos sistemas municipais.

Não pretendemos negar a relevância dos estudos em realidades microdinâmicas, visto que a condução da política de atenção primária seja atribuída aos municípios e que este, portanto, é capaz de responder aos resultados produzidos por estas investigações.

No entanto, cabe a necessidade de ampliar o escopo dos estudos no sentido de identificar as lacunas da política de forma abrangente, abarcando os elementos incomuns entre as realidades estudadas. E desta forma perseguir a renovação do conceito para transformação da prática e consequentemente transição efetiva do modelo assistencial.

Desde 2011 está em andamento o Programa Nacional de Melhoria do Acesso e da Qualidade da Atenção Básica (PMAQ), cujo objetivo principal é ampliar o acesso e melhorar a qualidade da Atenção Básica brasileira. O Programa está dividido em quatro fases e neste momento se prepara para o início da terceira delas, que consiste na avaliação externa das equipes de Atenção Básica, com observação da Unidade Básica de Saúde (estrutura), entrevista com os profissionais e verificação de documentos, entrevistas com os usuários, além de um módulo online que deve ser respondido por gestores e equipes com informações complementares aos módulos anteriores (MS, 2012).

O PMAQ é uma política direcionada a qualificação da gestão, cuja avaliação de desempenho das equipes está articulada a transferência de recursos aos municípios e unidades que aderiram ao programa. Independente disso espera-se um novo ápice na produção de estudos acerca da APS no Brasil a partir de 2014 em decorrência da condução do Programa. A possibilidade que se constrói com os resultados que serão disponibilizados é de avaliar a APS brasileira em todo território nacional, o que induz a realização de estudos de grande abrangência, com comparação entre municípios e regiões.

A percepção acerca das inúmeras realidades locais dadas a diversidade brasileira bem como a possibilidade de olhar para essa diversidade inserida no contexto nacional de implementação da APS, representa um novo momento para os estudos que abordam o tema.
Esperamos que com a emersão destes novos estudos possamos entender melhor os constrangimentos de cada cenário e, desta forma, caminhar no sentido de uma APS abrangente no Brasil, ancorada nos princípios do SUS e capaz de promover o cuidado orientado para a família, que atenda as demandas de cada comunidade.

Cabe ressaltar, no entanto, que o presente estudo não está imune às limitações inerentes aos estudos de revisão de literatura. Tanto no sentido de não poder assegurar que todos os estudos relevantes para o tema estejam disponíveis na base de dados escolhida para a pesquisa, quanto de garantir que a discussão realizada em cada artigo analisado traduza com comprometimento as realidades de cada contexto.

Ademais, a efervescência do tema, já referida anteriormente, faz crescer não somente as publicações de artigos em periódicos nacionais e internacionais - incluídos no escopo da presente análise -, mas também o crescimento de todo tipo de produção científica, por hora não analisados neste trabalho.

\section{Considerações Finais}

A APS assumiu novo protagonismo no discurso das agências internacionais de cooperação (OMS, 2005) e têm seu destaque reforçado na agenda política nacional há pelo menos dez anos ${ }^{44}$.

Com isso, podemos esperar um interesse entre os pesquisadores e pensadores da saúde pública em estudar os motivos dessa nova ascensão e as suas implicações na formulação de políticas orientadas para APS. Soma-se ao fato deste novo protagonismo reavivar elementos pertinentesà APS de cunho abrangente, referenciada pela primeira vez em Alma Ata e reorientada pelos organismos internacionais multilaterais em concepções seletivas e focalizadas.

Assim como se pretendeu com o presente estudo, percebe-se o crescente interesse pelo tema por parte dos pesquisadores nacionais devido ao aumento da produção evidenciada a partir do ano 2000. Com aumento gradativo ao longo da década, impulsionado a partir de 2007, ano seguinte a aprovação da Política Nacional de Atenção Básica.

Concomitante a este aumento esperado de publicações, vale ressaltar, a partir dos achados deste estudo, a aproximaçãodo referencial teórico 
que fundamenta a APS abrangente presente de forma predominante nos estudos analisados.

Entre os elementos enunciados tanto por Starfield (2002) quanto pela PNAB, a integração da APS a uma rede articulada de serviços de saúde é fundamental para efetivação da política. O número relevante de artigos cujos objetivos permeavam o estudo da integração evidencia a preocupação com esse constrangimento importante a efetivação da APS abrangente. Novos estudos, com vistas à análise destes resultados, se mostram de fundamental importância para compreendermos os processos que se impõe como limitadores e facilitadores da implementação da APS no Brasil.

O olhar direcionado para a política de APS brasileira no contexto macro político e do sistema de saúde ainda parece ser incipiente. $\mathrm{O}$ acúmulo de estudos acerca de realidades microdinâmicas é de suma importância para a compreensão do processo de implementação da política em um cenário onde se espera o mais alto grau de descentralização. Entretanto, priorizar este ponto de vista em detrimento de uma análise contextual mais ampla pode postergar a evidência de limites referentes à política.

Para entendermos os constrangimentos impostos a implementação e efetivação da política de APS de forma abrangente, que garanta aos usuários acesso, atenção integral e de qualidade em todos os níveis assistenciais, faz-se necessário a abordagem macro dinâmica do contexto e das relações que permeiam a política. Há de se transpor as limitações impostas pelas contradições inerentes à sociedade cujo modo de acumulação "coisifica" bens fundamentais da vida. Cabe a necessidade de reflexão, portanto, quanto à forma como pensamos o processo saúdedoença e qual o projeto de sociedade esperamos ao sugerirmos reformas - que promovem a manutenção do estado das coisas - e buscam corrigir as falhas que o mercado é incapaz de reparar.

Novas propostas conduzidas pelo Ministério da Saúde trazem indícios de que novos elementos para analisar a APS brasileira alimentarão o debate. As possibilidades que podem ser criadas através dos dados do PMAQ são inúmeras, de grande abrangência e permeiam os muitos olhares dos serviços de saúde gestores, profissionais, usuários. Os estudos acerca do tema supostamente aumentarão nos anos que se seguem sob influência do Programa e esperamos que com eles também aumentem o acesso e qualidade, sob os fundamentos da APS abrangente.

\section{Referências}

1. Shi L. Primary care, specialty care, and life chances. IntJ health serv 1994; 24:431-58.

2. Starfield B. Atenção Primária: equilíbrio entre necessidades de saúde, serviços e tecnologia. Brasília: UNESCO; Ministério da Saúde; 2002.

3. Viana ALD, Fausto MCR. Atenção Básica e Proteção Social: universalismo $x$ focalismo e espaço nãomercantil da assistência. In: Viana, ALD, Elias PEM,Ibañes N, organizadores. Proteção Social: dilemas e desafios. São Paulo: Hucitec;2005.p. 150-67.

4. Almeida C. Reforma de Sistemas de Saúde: tendências internacionais, modelos e resultados. In: Giovanella L, Escorel S, Lobato LVC, Noronha JC, Carvalho AI, organizadores. Políticas e Sistema de Saúde no Brasil. Rio de Janeiro:Fiocruz; 2012. p. 759-801.

5. GIOVANELLA, L. Atenção Primária à Saúde seletiva ou abrangente?Cad Saúde Pública 2008; 24(Supl1):727.

6. Mata JAS, Ruiz MLF. Definicion y caracterÌsticas de laatención primaria de salud. In: FADSP, organizador. SituacionActual y Futuro de laAtención Primaria de Salud. Madri: FADSP; 1993.

7. Fausto MCR, Matta GC. Atenção Primária à Saúde: histórico e perspectivas. In: Valéria MVGC, Corbo AAD, organizadores. Modelos de atenção e a saúde da família. Rio de Janeiro: EPSJV/FIOCRUZ; 2007.p. 43-67.

8. Escorel S, Nascimento DR, Edler FC. As origens da Reforma Sanitária e do SUS. In: Lima NT, Gerschman S, Edler FC, Suárez JM, organizadores. Saúde e Democracia: história e perspectivas do SUS. Rio de Janeiro: Fiocruz; 2005.p. 59-81.

9. Portaria $\mathrm{n}^{\mathrm{o}} 648$, de 28 de março de 2006. Aprova a Política Nacional de Atenção Básica, estabelecendo a revisão de diretrizes e normas para a organização da Atenção Básica para o Programa Saúde da Família (PSF) e o Programa Agentes Comunitários de Saúde (PACS). Diário Oficial da República Federativa do Brasil 2006; 29 mar.

10. Portaria $\mathrm{n}^{\mathrm{o}} 2.488$, de 21 de outubro de 2011. Aprova a Política Nacional de Atenção Básica, estabelecendo a revisão de diretrizes e normas para a organização da Atenção Básica, para a Estratégia Saúde da Família ESF e o Programa de Agentes Comunitários de Saúde - PACS. Diário Oficial da República Federativa do Brasil 2011; 24 out.

11. Baptista TWF, Mattos RA. Sobre Política (ou o que achamos pertinente refletir para analisar políticas). In: Mattos RA, Baptista TWF. Caminhos para análise das políticas de saúde. 2011. p.52-91.

12. Almeida PF, Giovanella L, Mendonça MHM, Escorel S. Desafios à coordenação dos cuidados em saúde: estratégias de integração entre níveis assistenciais em grandes centros urbanos. Cad Saúde Pública 2010; 26(2):286-98.

13. Almeida PF, Fausto MCR, Giovanella L. Fortalecimento da atenção primária à saúde: 
estratégia para potencializar a coordenação dos cuidados. Rev Panam Salud Publica 2011;29(2):84-95.

14. Assis EG, Beraldo AA, Monroe AA, Scatena LM, Cardozo-Gonzales RI, Palha PF et al. A coordenação da assistência no controle da tuberculose. Revesc de enf USP 2012; 46(1):111-8.

15. CardosoMO, Vieira-da-Silva LM. Avaliação da cobertura da atenção básica à saúde em Salvador, Bahia, Brasil (2000 a 2007). Cad Saúde Pública2012; 28(7):1273-84.

16. CufinoSvitone E, Garfield R, Vasconcelos MI, Craveiro VA. Primary health care lessons from the Northeast of Brazil: the Agentes de Saúde Program. Rev Panam Salud Publica 2000;7(5):293-302.

17. Cunha ABO, Vieira-da-Silva LM. Acessibilidade aos serviços de saúde em um município do Estado da Bahia, Brasil, em gestão plena do sistema.Cad Saúde Pública 2010; 26(4):725-37.

18. Delfini PSS, Sato MT, Antoneli PP. Parceria entre CAPS e PSF: o desafio da construção de um novo saber.Rev C S Col2009; 14(Supl1):1483-92.

19. Escorel S, Giovanella L, Mendonça MHM, Senna MCM. O Programa de Saúde da Família e a construção de um novo modelo para a atenção básica no Brasil. Rev Panam Salud Publica 2007; 21(2):16476.

20. Gaíva MA, Silva AMN. O processo de implantação do Programa Saúde da Família no Estado de Mato Grosso, Brasil: perspectiva de atores sociais da gestão estadual. CogitareEnferm 2008;13(4):576-84.

21. Gil CRR. Atenção primária, atenção básica e saúde da família: sinergias e singularidades do contexto brasileiro. Cad Saúde Pública 2006; 22(6):1171-81.

22. Giovanella L, Mendonça MHM, Almeida PF, Escorel S, Senna MCM, Fausto MCR et al. Saúde da família: limites e possibilidades para uma abordagem integral de atenção primária à saúde no Brasil. RevC S Col 2009; 14(3):783-94.

23. Gomes FM, Silva MGC. Programa Saúde da Família como estratégia de atenção primária: uma realidade em Juazeiro do Norte. Rev C S Col 2011; 16(Supl. 1):893-902.

24. Gomes KO de, Cotta RMM, Araújo RMA, Cherchiglia ML, Martins TCP. Atenção Primária à Saúde a "menina dos olhos" do SUS: sobre as representações sociais dos protagonistas do Sistema Único de Saúde. Rev C S Col 2011; 16(Supl. 1):881-92.

25. Guedes HM, Paula LD, Nakatani AYK, Coelho AB. Resultados alcançados com a estratégia saúde da família após cinco anos de implantação em uma cidade do interior de Minas Gerais. Rev Min Enf 2007; 11(4):363-8.

26. Heimann LS, Ibanhes LC, Boaretto RC, Castro IEN, Telesi Júnior E, Cortizo CT et al. Atenção primária em saúde: um estudo multidimensional sobre os desafios e potencialidades na Região Metropolitana de São Paulo (SP, Brasil).Rev C S Col 2011; 16(6):2877-87.

27. Lavras C. Atenção Primária à Saúde e a Organização de Redes Regionais de Atenção à Saúde no Brasil. Saúde Soc2011; 20(4):867-74.

28. Leão CDA, Caldeira AP. Avaliação da associação entre qualificação de médicos e enfermeiros em atenção primária em saúde e qualidade da atenção. Rev C S Col 2011; 16(11):4415-23.

29. Marsiglia RMG. Universalização do acesso ao Sistema Único de Saúde no Brasil: desafios para a Atenção
Primária à Saúde. CadTerOcup UFSCar 2012; 20(3): 317-25.

30. Medina MG, Hartz ZMA. The role of the Family Health Program in the organization of primary care in municipal health systems. CadSaúdePública 2009; 25(5):1153-67.

31. Mendonça CS. Saúde da Família, agora mais do que nunca! Rev C S Col 2009; 14(Supl1):1493-7.

32. Mitre SM, Andrade EIG, Cotta RMM. Avanços e desafios do acolhimento na operacionalização e qualificação do Sistema Único de Saúde na Atenção Primária: um resgate da produção bibliográfica do Brasil. Rev C S Col 2012; 17(8):2071-85.

33. Motta LB, Aguiar AC, Caldas CP. Estratégia Saúde da Família e a atenção ao idoso: experiências em três municípios brasileiros. Cad Saúde Pública 2011; 27(4):779-86.

34. Onocko-Campos RT, Campos GWS, Ferrer AL, Corrêa CRS, Madureira PR, Gama CAP et al. Avaliação de estratégias inovadoras na organização da Atenção Primária à Saúde. Rev Saúde Pública 2012;46(1):43-50.

35. Souza MF, Hamann EM. Programa Saúde da Família no Brasil: uma agenda incompleta? RevC S Col 2009, 14(Supl1):1325-35.

36. Souza MLP, Garnelo L. "É muito dificultoso!": etnografia dos cuidados a pacientes com hipertensão e/ou diabetes na atenção básica, em Manaus, Amazonas, Brasil.Cad Saúde Pública 2008; 24(Sup11):91-9.

37. Tavares MFL, Mendonça MHM, Rocha RM.Práticas em saúde no contexto de reorientação da atenção primária no Estado do Rio de Janeiro, Brasil, na visão das usuárias e dos profissionais de saúde. Cad Saúde Pública 2009; 25(5):1054-62.

38. Tomasi E, Facchini LA, Thumé E, Piccini RX, Osorio A, Silveira DS et al. Características da utilização de serviços de atenção básica à saúde nas regiões Sul e Nordeste do Brasil: diferenças por modelo de atenção. RevC S Col 2011;16(11):4395-404.

39. Trad, LAB Castellanos MEP Guimarães MCS da.Acessibilidade à atenção básica a famílias negras em bairro popular de Salvador, Brasil. Rev Saúde Pública 2012;46(6):1007-13.

40. Villela WV, Araújo EC, Ribeiro AS, Cuginotti AP, Hayana ET, Brito FC et al. Desafios da Atenção Básica em Saúde: a experiência de Vila Mariana, São Paulo, Brasil. Cad Saúde Pública 2009; 25(6):1316-324.

41. Conill EM, Fausto MCR. Análisis de laIntegración de laAtención Primaria emSalud em laRed de Servicios em Europa y América Latina. ProyectoEUROsociALSalud;2009.Intercambio "Fortalecimiento de laIntegración de laAtenciónPrimaria com otros Niveles de Atención" [documento técnico]. Rio de Janeiro:IRD;[acessado 2012 Mar 29]. Disponível em:http:/ / biblioteca.programaeurosocial.eu/PDF/ Salud/Salud11.pdf.

42. Viana ALD, Dal Poz MR. A Reforma do Sistema de Saúde no Brasil e o Programa de Saúde da Família. PHYSIS: Rev. Saúde Coletiva 2005; 15(Supl1):225- 64.

43. Carvalho JA, Medina MG. A produção bibliográfica sobre o Programa de Saúde da Família no Brasil: análise bibliométrica do período 1994-200 [tese]. Bahia: Instituto de Saúde Coletiva, UFBA; 2010. 
44. Castro ALB de, Machado CV. A política federal de atenção básica à saúde no Brasil nos anos 2000. PHYSIS: Rev. Saúde Coletiva 2010;22(2):477-506. 